九州大学学術情報リポジトリ

Kyushu University Institutional Repository

\title{
Autopsy case of later-onset pontocerebellar hypoplasia type 1 : Pontine atrophy and pyramidal tract involvement
}

Sanefuji, Masafumi

Department of Pediatrics, Graduate School of Medical Sciences, Kyushu University

Kira, Ryutaro

Department of Pediatrics, Graduate School of Medical Sciences, Kyushu University

Matsumoto, Kenichi

Department of Neuropathology, Graduate School of Medical Sciences, Kyushu University

Gondo, Kenjiro

Department of Pediatrics, Graduate School of Medical Sciences, Kyushu University

他

http://hdl. hand le. net/2324/26028

出版情報：Journal of Child Neurology. 25 (11)，pp.1429-1434，2010-11. SAGE バージョン:

権利関係 : (C) 2010 by SAGE Publications 
Title: Autopsy case of later-onset pontocerebellar hypoplasia type 1: pontine atrophy and pyramidal tract involvement

Running title: later-onset pontocerebellar hypoplasia type 1

Authors: Masafumi Sanefuji MD, $\mathrm{PhD}^{1}$, Ryutaro Kira MD, $\mathrm{PhD}^{1}$, Kenichi Matsumoto $\mathrm{MD}, \mathrm{PhD}^{2}$, Kenjiro Gondo $\mathrm{MD}, \mathrm{PhD}^{1}$, Hiroyuki Torisu $\mathrm{MD}, \mathrm{PhD}^{1}$, Hideshi Kawakami $\mathrm{MD}, \mathrm{PhD}^{3}$, Toru Iwaki MD, $\mathrm{PhD}^{2}$, Toshiro Hara $\mathrm{MD}, \mathrm{PhD}^{1}$

\section{Affiliations:}

${ }^{1}$ Department of Pediatrics, Graduate School of Medical Sciences, Kyushu University 3-1-1 Maidashi, Higashi-ku, Fukuoka 812-8582, Japan

Tel: +81-92-642-5421, Fax: +81-92-642-5435

${ }^{2}$ Department of Neuropathology, Graduate School of Medical Sciences, Kyushu University

3-1-1 Maidashi, Higashi-ku, Fukuoka 812-8582, Japan.

Tel: +81-92-642-5421, Fax: +81-92-642-5435

${ }^{3}$ Department of Epidemiology, Division of Bio-Medical Informatics, Research Institute for Radiation Biology and Medicine, Hiroshima University

1-2-3 Kasumi, Minami-ku, Hiroshima 734-8553, Japan

Tel: +81-82-257-5846, Fax: +81-82-257-5848

Masafumi Sanefuji: the main attending physician, who wrote the manuscript. e-mail: sane26@pediatr.med.kyushu-u.ac.jp 
Ryutaro Kira: the prior attending physician of the patient who had long clinical courses. e-mail: kirarin@tb3.so-net.ne.jp

Kenichi Matsumoto: the doctor who anatomize the patient e-mail: matsumo@kyushu-ctr-hsp.com Current affiliation: Department of neurosurgery, Kyushu Central Hospital 3-23-1 Shiobaru, Minami-ku, Fukuoka 815-8588, Japan Tel: +81-92-541-4936, Fax: +81-92-541-4540

Kenjiro Gondo: the prior attending physician e-mail: kenjirogondo@mac.com Current affiliation: Department of Pediatric Neurology, Fukuoka Children's Hospital and Medical Center for Infectious Diseases

2-5-1 Tojin-machi, Chuo-ku, Fukuoka 810-0063, Japan

Tel: +81-92-713-3111, Fax: +81-92-713-3120

Hiroyuki Torisu: the second attending physician e-mail: htorys@pediatr.med.kyushu-u.ac.jp

Hideshi Kawakami: the doctor who performed the DNA analysis e-mail: hkawakam@hiroshima-u.ac.jp 
e-mail: iwaki@np.med.kyushu-u.ac.jp

Toshiro Hara: the professor who examined the patient and wrote the manuscript e-mail: harat@pediatr.med.kyushu-u.ac.jp

Correspondence: Masafumi Sanefuji

Department of Pediatrics, Graduate School of Medical Sciences, Kyushu University, 3-1-1 Maidashi, Higashi-ku, Fukuoka 812-8582, Japan Tel: +81-92-642-5421, Fax: $+81-92-642-5435$.

e-mail: sane26@pediatr.med.kyushu-u.ac.jp

This work was supported in part by a Grant-in-Aid for Scientific Research from the Ministry of Education, Culture, Sports, Science and Technology of Japan. The authors have no conflicts of interest to declare.

Word counts: text 1687, references 463, figure legends 228

Abbreviations: CT: computed tomography, MRI: magnetic resonance imaging

Key words: pontocerebellar hypoplasia, anterior horn cell, child 


\begin{abstract}
The combination of pontocerebellar hypoplasia and anterior horn cell degeneration is classified as pontocerebellar hypoplasia type 1. Although most cases exhibit severe muscle weakness and hypotonia neonatally with short life spans, some cases exhibit a later onset with a longer life span and show cerebellar atrophy without pontine involvement. We present a child who exhibited neurological deterioration and progressive atrophy of the cerebellum and pons, with onset of symptoms at 20 months and the death at 15 years of age. The pathological findings disclosed anterior horn cell degeneration and pyramidal tract involvement in addition to pontocerebellar atrophy, leading to the diagnosis of pontocerebellar hypoplasia type 1 . The present case suggests that the degenerative pattern of later-onset pontocerebellar hypoplasia type 1 is similar to that of prenatal-onset cases. Further reports of later-onset cases with histopathological examination are required to elucidate the nosology and etiology of the disorder.
\end{abstract}




\section{INTRODUCTION}

Pontocerebellar hypoplasias are a group of prenatal-onset neurodegenerative disorders showing hypoplasia or atrophy of the cerebellum and pons, with severe developmental delay, mental retardation, and often progressive courses ${ }^{1}$. Among six clinical subtypes of pontocerebellar hypoplasias, the combination of pontocerebellar hypoplasia and anterior horn cell degeneration is exceptionally rare and is classified as pontocerebellar hypoplasia type $1^{2}$, which is also called pontocerebellar hypoplasia with infantile spinal muscular atrophy. The phenotype is characterized by severe muscle weakness and hypotonia starting prenatally or at birth, with a life span not exceeding a few months in most cases ${ }^{3}$.

However, it has been found that some cases of pontocerebellar hypoplasia type 1 exhibit later onsets from several months to 2 years, and longer life spans from 2 to 11 years ${ }^{2,}{ }^{4-6}$. In these later-onset cases, magnetic resonance imaging (MRI) and pathological findings revealed anterior horn cell degeneration and cerebellar atrophy but a preserved pons, and provided no evidence of spinal cord involvement.

We present a child who exhibiting neurological deterioration and progressive atrophy of the cerebellum and pons, with the onset of symptoms at 20 months and the death at 15 years of age. The pathological findings disclosed the anterior horn cell degeneration and the pyramidal tract involvement in addition to the pontocerebellar atrophy, leading to the diagnosis of pontocerebellar hypoplasia type 1 .

\section{CASE REPORT}

The patient was the third child of healthy nonconsanguineous parents. His elder brothers were normal. Pregnancy was uneventful and he was born at full term with a 
birth weight of 2,830g. He gained head control by 4 months and was sitting by 7 months. He stood with support by 14 months, which could be considered retrospectively as the first sign of delayed motor milestones. He could speak several words at 18 months. At the age of 20 months, he was examined because he could not stand without support. Initial diagnoses of cerebral palsy and Klinefelter's syndrome were made because the patient's karyotype was 47, XXY. He showed developmental regression after 2 years old: loss of standing with support at 2 years, sitting at 3 years, and speech at 4 years. He also had slowly progressive spasticity and muscular atrophy from the lower extremities to the upper extremities. At 4 years, a sural nerve biopsy revealed no abnormalities. At 5 years, the patient's first epileptic seizures occurred, and the first sign of ophthalmoplegia, i.e., exotropia in the left eye, was noticed. An electroencephalogram showed slow background activity and epileptiform discharges. Computed tomography (CT) of the brain showed mild ventricular dilatation and probable cerebellar atrophy (Figure 1A). At 9 years, fundoscopy revealed no abnormalities. Neurophysiological tests were also performed as follows. A right median nerve conduction study showed normal velocities but reduced compound muscle action potential, suggesting neuronopathy or axonopathy, and auditory evoked brainstem responses showed only wave I on both sides. MRI of the brain revealed moderate ventricular dilatation and moderate atrophy of the cerebellum (Figure 1C and 1D). The following investigations were all normal: blood counts, serum biochemistry, thyroid function, serum ceruloplasmin, serum amino acids, urine organic acids and amino acids. Cerebrospinal fluid examination showed no abnormality: leukocytes and levels of protein, glucose, lactate and pyruvate. There were no triplet repeat expansions in the genes of spinocerebellar ataxia types 1, 2, 3, 6 and 7, and dentatorubral-pallidoluysian atrophy. 
Semiquantitative PCR analysis demonstrated two copies of the proteolipid protein 1 gene, concurrent with the two X chromosomes of Klinefelter's syndrome. All of the voluntary eye movements were completely restricted with bilateral exophthalmos by the age of 13 years. There was no tongue fasciculation. The patient could only recognize simple instructions such as putting his hands up. He exhibited prominent muscular atrophy, marked stiffness and contracture of the extremities, and severe scoliosis. A tracheostomy was performed at 14 years because he had respiratory difficulties with recurrent infections. Brain CT revealed severe atrophy of the cerebellum and the pons (Figure 1B). The patient died from respiratory failure at 15 years.

Autopsy findings

General autopsy revealed scoliosis, esophageal erosion, pleural and peritoneal effusion, and testicular atrophy, which was compatible with Klinefelter's syndrome. The formalin-fixed brain weighed $970 \mathrm{~g}$. The cerebral hemispheres were well-formed. There were severe atrophies of the cerebellum, brain stem, spinal cord and anterior roots (Figure 2). Histological examination revealed neuronal loss with gliosis in the internal segments of the globus pallidus, lateral nuclei of the thalamus, subthalamic nucleus, red nucleus, cerebellar granule cells, Purkinje cells (Figure 3A), and the fastigial and dentate nuclei (Figure 2B and 3B) of the cerebellum. Neuronal loss was also observed in the inferior olivary, vestibular and hypoglossal nuclei (Figure 2C and 3C). Mild neuronal loss was observed in the abducens, facial and pontine nuclei. Degeneration of the superior and inferior cerebellar peduncles and central tegmental tracts was observed. Degeneration of the pyramidal tracts was evident in the cerebral peduncle, pons, and 
medulla oblongata (Figure 2C). In the spinal cord, there was neuronal loss of anterior horn cells (Figure 3D) and diffuse degeneration of the anterior and lateral funiculi including the pyramidal and spinocerebellar tracts (Figure 2D). No neuronal inclusions were identified by immunostaining with antibodies to polyglutamine and ubiquitin. In summary, there was degeneration of the vestibulocerebellar tracts, the afferent and efferent tracts of the cerebellum, the subthalamic nucleus - globus pallidus system, and the upper and lower motor neuron system.

\section{DISCUSSION}

The present case exhibited infantile-onset motor and cognitive deterioration showing prominent muscular atrophy and spasticity, with progressive atrophy of the cerebellum and pons. Neuropathological findings revealed pontocerebellar atrophy, anterior horn cell degeneration and severe pyramidal tract involvement.

The combination of pontocerebellar atrophy and anterior horn cell degeneration has been previously reported as cerebellar hypoplasia in Werdnig-Hoffmann disease ${ }^{7}$, infantile olivopontocerebellar atrophy plus spinal muscular atrophy ${ }^{8}$, or spinal muscular atrophy plus olivopontocerebellar atrophy ${ }^{9}$. In 1993, Barth called this combination pontocerebellar hypoplasia type $1^{3}$. Linkage to the survival of motor neuron 1 gene at 5q12, the cause of classical spinal muscular atrophy, has been excluded ${ }^{5}$. Recently, a nonsense mutation in the vaccinia-related kinase 1 gene was identified in a consanguineous family ${ }^{2}$.

Pontocerebellar hypoplasia type 1 may be a heterogenous disorder and shows considerable variability in clinical and pathological features ${ }^{10}$. The clinical manifestation includes hypotonia, areflexia, congenital contractures, respiratory and 
feeding difficulties, seizures, nystagmus, and no gross motor development. The pathological findings consist of atrophy or gliosis of the cerebellum, pons, olives, thalami and pallidum, with granular and Purkinje cell loss, and degeneration of the inferior olivary and dentate nuclei, and anterior horn cells. Most of the cases show clinical manifestations neonatally, but some cases exhibit later onsets and more benign but progressive clinical courses. In 2003, three cases with later-onset pontocerebellar hypoplasia type 1 were reported. The onsets were in the first year of life and life spans were 2-4 years ${ }^{4}$. Recently, two siblings were reported, exhibiting later-onset pontocerebellar hypoplasia type 1 with cerebellar atrophy and anterior horn cell involvement demonstrated by brain MRI and muscle biopsy, respectively. The onsets were 1 and 2 years of age, and the siblings showed slow neurodegenerative clinical courses that included cognitive and motor functions; they are still alive at 6 and 9 years.

The name "pontocerebellar hypoplasia" may have to be reconsidered because the process is mainly atrophy rather than hypoplasia (primary defect) and the pons is not necessarily involved. Several cases with pontocerebellar hypoplasia type 1 have exhibited progressive deterioration with fetal onset ${ }^{10-12}$ or infantile onset ${ }^{6}$, rather than static developmental delay. It was proposed that the conditions described as pontocerebellar hypoplasia should be more accurately termed pontocerebellar atrophies based on serial neuroimaging studies ${ }^{1}$. In later-onset pontocerebellar hypoplasia type 1 , neuroimaging and pathological findings have demonstrated a preserved pons with cerebellar atrophy ${ }^{4,5}$. In our case, cerebellar atrophy progressed from 5 to 14 years, and pontine atrophy was demonstrated for the first time at 14 years. The reason for the pontine atrophy in our later-onset case may be due to the longer clinical course of approximately 13 years than those of the previously reported cases of approximately 
2-4 years. The pons may be less vulnerable than the cerebellum, and its atrophy may emerge when the disease has progressed further.

The involvement of the pyramidal tract is not a cardinal feature of pontocerebellar hypoplasia type 1 but previous reports have described this finding. Several cases have shown pyramidal tract signs such as Babinski signs, increased muscle tonus, brisk deep tendon reflex, and ankle and patellar clonus ${ }^{2,4-6,11,12}$, although it is not clear whether these pyramidal tract signs are due to the cerebrum or spine. A neuropathological report of a case considered as prenatal-onset pontocerebellar hypoplasia type 1 showed involvement of the lateral and ventral white matter including the corticospinal tract ${ }^{13}$, which is similar to our case. The pyramidal tract could be one of the degenerative lesions in later-onset pontocerebellar hypoplasia type 1.

To our knowledge, an association of pontocerebellar hypoplasia with Klinefelter's syndrome has not been reported, but other similar disorders such as spinocerebellar ataxias are reported to be accompanied by the abnormal karyotype ${ }^{14-16}$. However, the phenotypes are not distinct from other spinocerebellar ataxias. In our case, the association of pontocerebellar hypoplasia and Klinefelter's syndrome may have been coincidental because the occurrence rate of Klinefelter's syndrome is relatively high, with one in 500-1,000 male births ${ }^{17,18}$. However, androgen insufficiency accompanied by Klinefelter's syndrome might play a part in the pathogenesis because androgens affect the morphology and the survival of spinal and brainstem motor neurons in vivo ${ }^{19-21}$.

In conclusion, the present case suggests that later-onset pontocerebellar hypoplasia type 1 can exhibit not only cerebellar atrophy and anterior horn cell degeneration but also pontine atrophy and pyramidal tract involvement, similar to 
prenatal-onset cases, as the disease progresses extremely. Further reports of later-onset cases with histopathological examination are required to elucidate the nosology and etiology of the disorder.

Acknowledgments: We thank Dr. Akiko Iwaki for the analysis of the proteolipid protein 1 gene. This work was supported in part by a Grant-in-Aid for Scientific Research to Masafumi Sanefuji from the Ministry of Education, Culture, Sports, Science and Technology of Japan. 


\section{FIGURE LEGENDS}

Figure 1. Serial neuroimaging from 5 to 14 years of age. A, Brain computed tomography $(\mathrm{CT})$ shows probable cerebellar atrophy with a preserved pons at 5 years. $\mathbf{B}$, Brain CT shows obvious atrophy of the cerebellum and pons at 14 years. C, D, T2-weighted axial (C) and T1-weighted sagittal (D) slices of brain magnetic resonance imaging (MRI) show cerebellar atrophy without apparent involvement of the pons at 9 years.

Figure 2. Macrographs of the brain, cerebellum and cervical cord. Marked atrophy of the pons and cerebellum is observed in the basal view (A). A part of the right frontal lobe was resected at the autopsy. $\mathbf{B}$, The efferent tracts of the cerebellum and dentate nucleus are severely degenerated. C, The pyramids of medulla oblongata are severely degenerated and neuronal loss is observed in the inferior olivary nucleus. D, The anterior roots and the anterior and lateral funiculi of the cervical cord are severely degenerated, while the posterior roots and posterior funiculus are well preserved. B-D: Klüver-Barrera stain.

Figure 3. Degeneration of the cerebellar (A and B) and motor systems (C and D). Light micrographs show loss of Purkinje cells (A), severe gliosis of the dentate nucleus (B), loss of neurons in the hypoglossal nucleus (C), and loss of motor neurons in the anterior horn of the spinal cord (D). Hematoxylin and eosin stain, $\times 400$. 


\section{REFERENCES}

1. Parisi MA, Dobyns WB. Human malformations of the midbrain and hindbrain: review and proposed classification scheme. Mol Genet Metab. 2003;80(1-2):36-53.

2. Renbaum P, Kellerman E, Jaron R, et al. Spinal muscular atrophy with pontocerebellar hypoplasia is caused by a mutation in the VRK1 gene. Am J Hum Genet. 2009;85(2):281-289.

3. Barth PG. Pontocerebellar hypoplasias. An overview of a group of inherited neurodegenerative disorders with fetal onset. Brain Dev. 1993;15(6):411-422.

4. Rudnik-Schoneborn S, Sztriha L, Aithala GR, et al. Extended phenotype of pontocerebellar hypoplasia with infantile spinal muscular atrophy. Am J Med Genet A. 2003;117A(1):10-17.

5. Muntoni F, Goodwin F, Sewry C, et al. Clinical spectrum and diagnostic difficulties of infantile ponto-cerebellar hypoplasia type 1. Neuropediatrics. 1999;30(5):243-248.

6. Lev D, Michelson-Kerman M, Vinkler $\mathrm{C}$, et al. Infantile onset progressive cerebellar atrophy and anterior horn cell degeneration--a late onset variant of PCH-1? Eur J Paediatr Neurol. 2008;12(2):97-101.

7. Norman RM. Cerebellar hypoplasia in Werdnig-Hoffmann disease. Arch Dis Child. 1961;36:96-101.

8. Chou SM, Gilbert EF, Chun RW, et al. Infantile olivopontocerebellar atrophy with spinal muscular atrophy (infantile OPCA + SMA). Clin Neuropathol. 1990;9(1):21-32.

9. Rudnik-Schoneborn S, Forkert R, Hahnen E, et al. Clinical spectrum and diagnostic criteria of infantile spinal muscular atrophy: further delineation on the basis of SMN gene deletion findings. Neuropediatrics. 1996;27(1):8-15.

10. Ryan MM, Cooke-Yarborough CM, Procopis PG, et al. Anterior horn cell disease and olivopontocerebellar hypoplasia. Pediatr Neurol. 2000;23(2):180-184.

11. Goutieres F, Aicardi J, Farkas E. Anterior horn cell disease associated with pontocerebellar hypoplasia in infants. $J$ Neurol Neurosurg Psychiatry. 1977;40(4):370-378.

12. Salman MS, Blaser S, Buncic JR, et al. Pontocerebellar hypoplasia type 1: new leads for an earlier diagnosis. J Child Neurol. 2003;18(3):220-225.

13. Steiman GS, Rorke LB, Brown MJ. Infantile neuronal degeneration masquerading as Werdnig-Hoffmann disease. Ann Neurol. 1980;8(3):317-324.

14. Hecht A, Ruskin H. Seminiferous tubule dysgenesis (Klinefelter's syndrome) 
associated with familiar cerebellar ataxia. $J$ Clin Endocrinol Metab. 1960;20:1184-1190.

15. Indemini M, Ammann F. Spinocerebellar hereditary degeneration (SCHD) associated with the Klinefelter syndrome. Confin Neurol. 1963;23:155-164.

16. Stanzani P, Bruno L, Pollari Maglietta E, et al. A case of heredo-ataxia in a subject with karyotype 49 XXXXY. Brain Dev. 1986;8(5):570-571.

17. Nielsen J, Wohlert M. Chromosome abnormalities found among 34,910 newborn children: results from a 13-year incidence study in Arhus, Denmark. Hum Genet. 1991;87(1):81-83.

18. Nielsen J, Sillesen I. Incidence of chromosome aberrations among 11148 newborn children. Humangenetik. 1975;30(1):1-12.

19. Brooks BP, Merry DE, Paulson HL, et al. A cell culture model for androgen effects in motor neurons. J Neurochem. 1998;70(3):1054-1060.

20. Forger NG, Hodges LL, Roberts SL, et al. Regulation of motoneuron death in the spinal nucleus of the bulbocavernosus. J Neurobiol. 1992;23(9):1192-1203.

21. Perez J, Kelley DB. Trophic effects of androgen: receptor expression and the survival of laryngeal motor neurons after axotomy. $J$ Neurosci. 1996;16(21):6625-6633. 
Figure 1
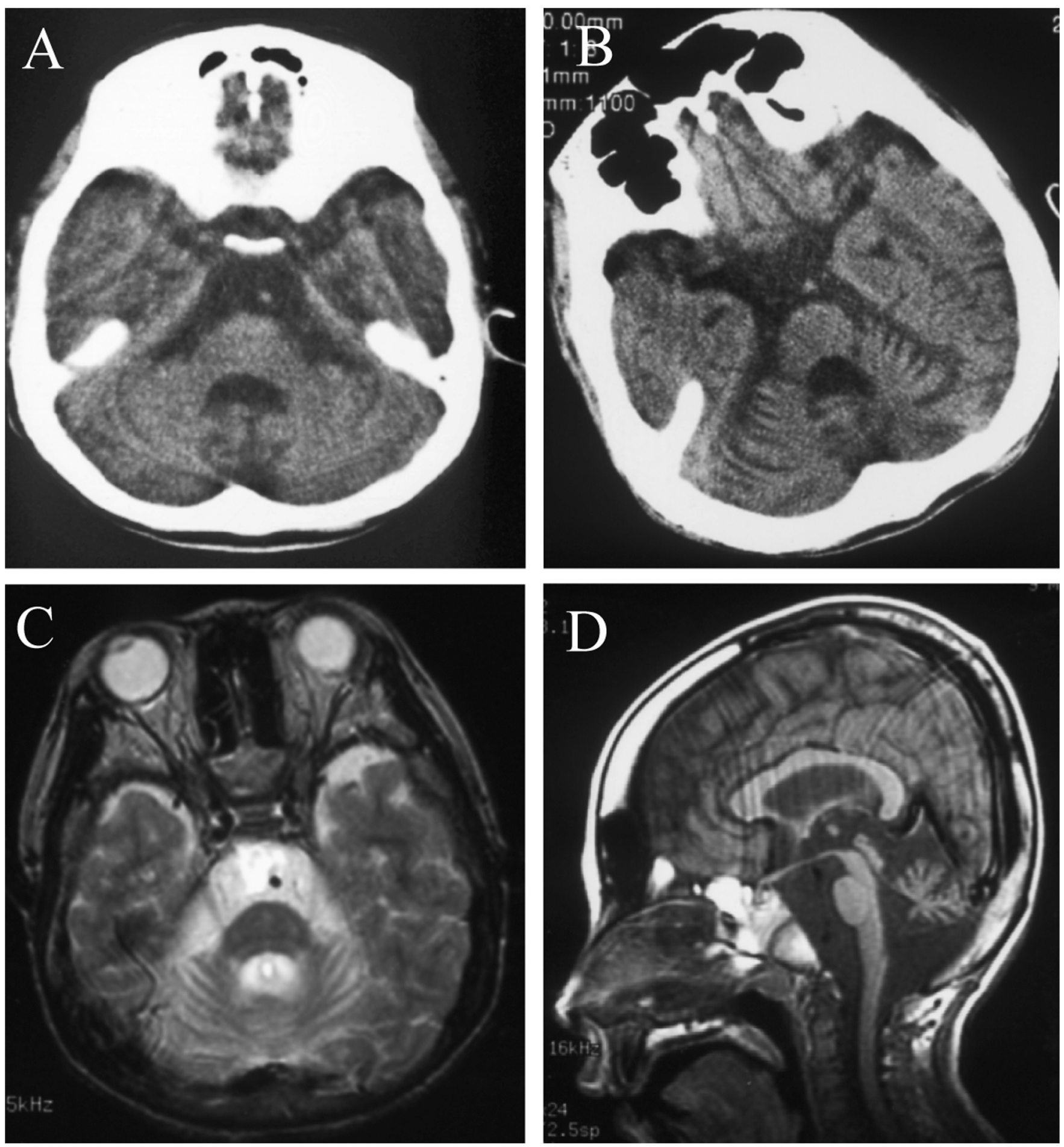
Figure 2
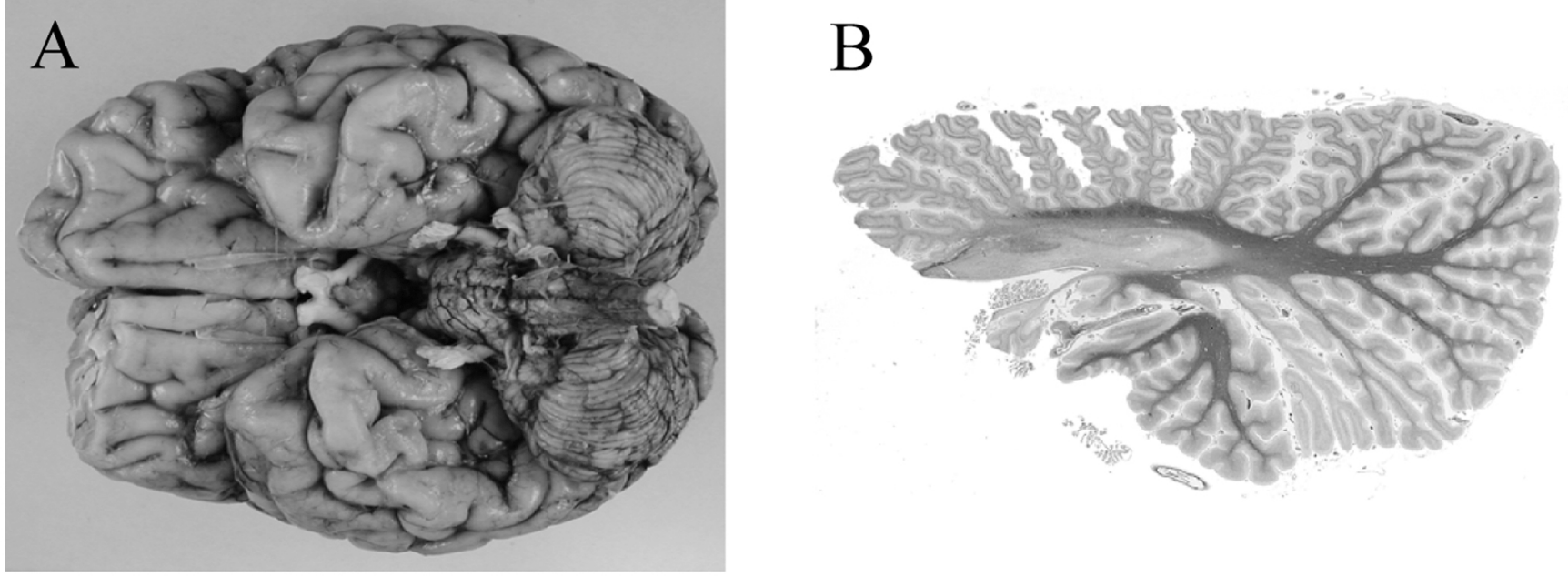

C

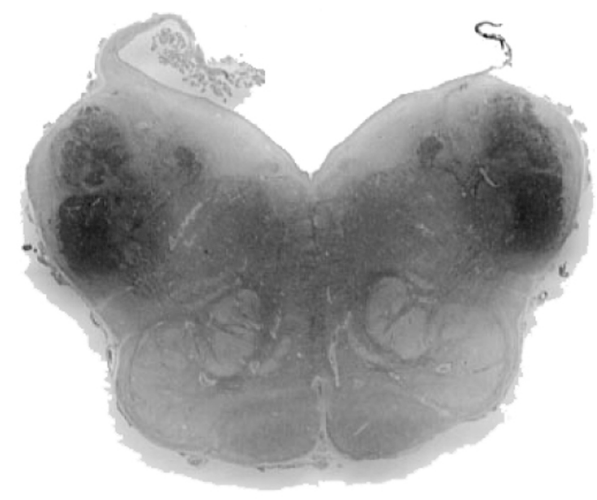

D

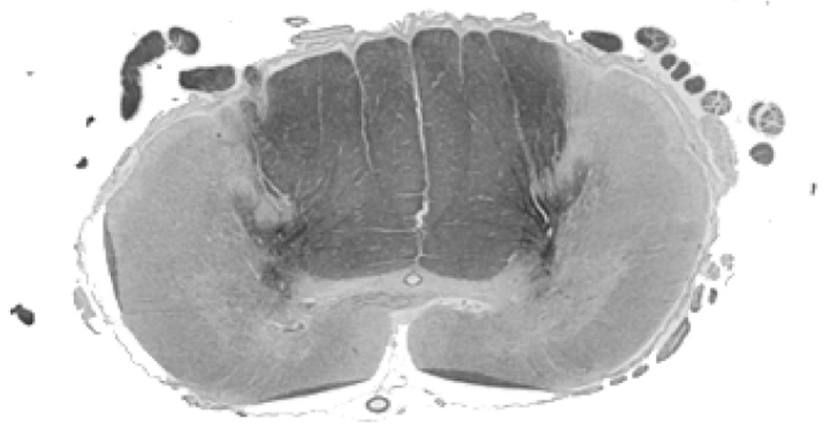


Figure 3
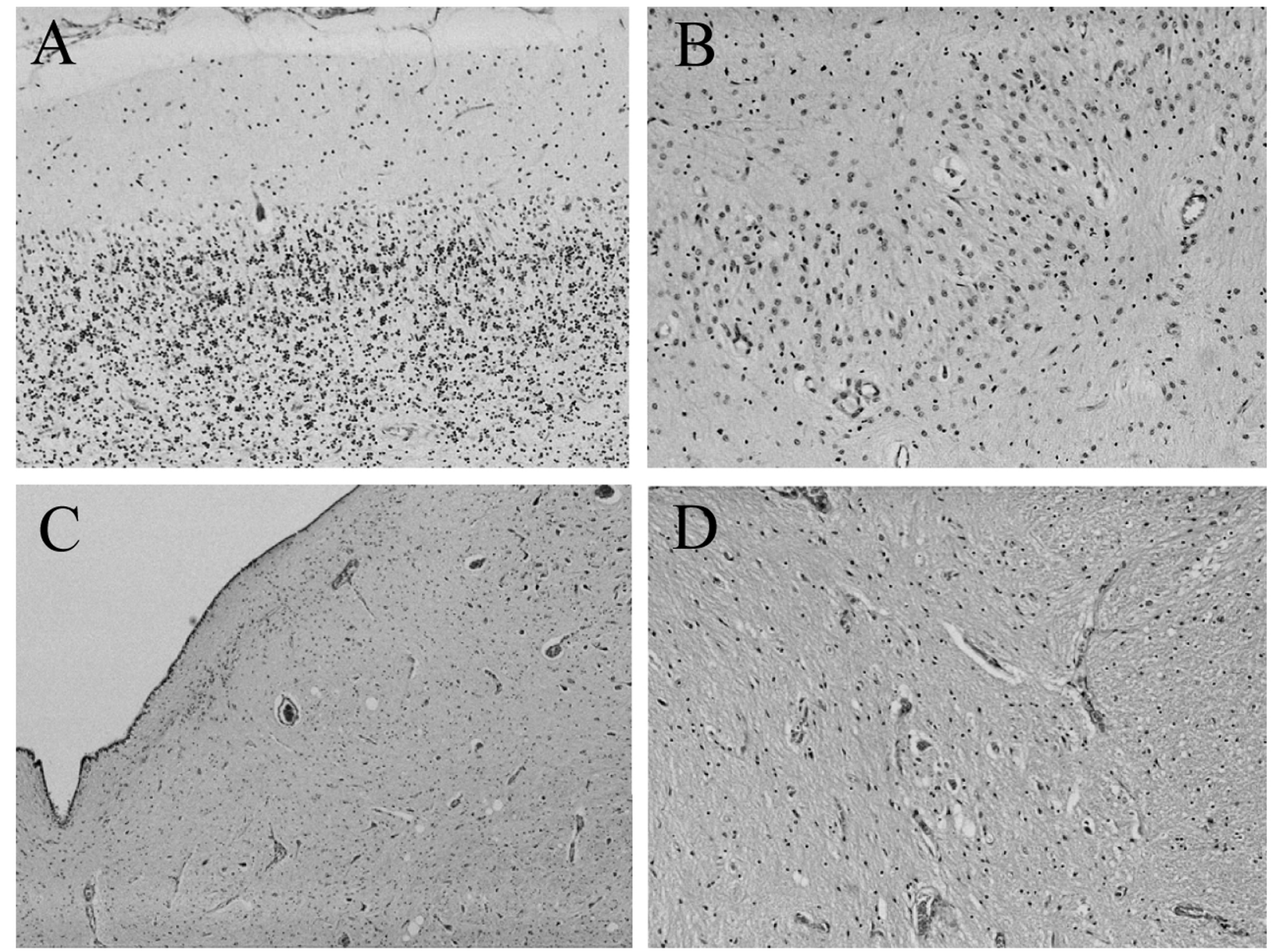